\title{
Civilisations
}

Revue internationale d'anthropologie et de sciences

humaines

56 | 2007

Après la catastrophe

\section{Après la catastrophe : mémoire, transmission et vérité dans les témoignages de rescapés des camps de concentration et d'extermination nazis}

Barbara Pirlot

\section{OpenEdition}

Journals

Édition électronique

URL : http://journals.openedition.org/civilisations/86

DOI : $10.4000 /$ civilisations. 86

ISSN : 2032-0442

\section{Éditeur}

Institut de sociologie de l'Université Libre de Bruxelles

\section{Édition imprimée}

Date de publication : 1 décembre 2007

Pagination : 21-41

ISBN : 2-87263-016-3

ISSN : 0009-8140

\section{Référence électronique}

Barbara Pirlot, «Après la catastrophe : mémoire, transmission et vérité dans les témoignages de rescapés des camps de concentration et d'extermination nazis », Civilisations [En ligne], 56 | 2007, mis en ligne le 01 décembre 2010, consulté le 30 avril 2019. URL : http://journals.openedition.org/ civilisations/86 ; DOI : 10.4000/civilisations.86 


\section{Après la catastrophe \\ Mémoire, transmission et vérité dans les témoignages de rescapés \\ des camps de concentration et d'extermination nazis}

Barbara PIRLOT

Résumé : Dans cet article, nous évoquerons la question des témoignages de la Shoah et des circonstances de leur énonciation. A cet effet, nous décrirons cet objet sociodiscursif particulier qu'est le témoignage, en détaillant ses composantes définitoires (certification autobiographique, pacte de référentialité, portée collective, etc.), en réfléchissant sur les conditions de son accréditation et en le comparant aux autres modalités et stratégies discursives (discours de l'historien, récit littéraire, etc.) utilisées pour transmettre cette réalité extrême et non partagée de la déportation et de l'expérience concentrationnaire.

Mots-clefs : témoignages de la Shoah, transmission, accréditation, discours.

Summary: In this paper, I focus on the question of the testimonies of the Shoah and the circumstances of their creation. The testimony, a specific sociodiscursive object, is described by detailling its defining elements (autobiographical certification, referentiality pact, collective thrust, etc.), by reflecting on the conditions of its accreditation and by comparing it to the other discursive modalities and strategies (the discourse of the historian, literary accounts, etc.) used to convey the extreme and unshared reality of deportation and the experience of concentration camps.

Keywords: testimony, Shoah, transmission, discourse, accreditation. 
Q ue reste-t-il après la catastrophe ? Que subsiste-t-il des événements tragiques de cette moitié du $20^{\mathrm{e}}$ siècle qui ont mené à l'extermination planifiée, rationalisée et industrialisée de plus de six millions d'êtres humains ${ }^{1}$, aujourd'hui, soixante ans après la libération des camps, alors que le nombre des rescapés encore en vie - mémoire-vive du drame - s'étiole et que, comme pour combler ce vide et relayer le phénomène de remémoration ${ }^{2}$, les commémorations s'enchaînent dans un effort quasi compulsif? A l'aube du $21^{\mathrm{e}}$ siècle, il convient donc de s'interroger avec plus d'acuité encore sur le passage du témoin, sur la manière la plus appropriée de rendre compte de ces événements extrêmes et sur la transmission de leur mémoire. Car, pour reprendre les mots de Pierre Mertens, "plus le temps s'écoule après Auschwitz, plus il s'avère indispensable de le reparler» (Mertens $2003: 51)$.

Des faits du passé, il subsiste deux types de traces : les traces matérielles (vestiges, documents, etc.) et les traces mnésiques. A partir de ces traces, et pour rendre compte de ce qui est réellement advenu dans le passé, s'articulent deux principales modalités discursives : le témoignage et le discours historique. Avec les récits des rescapés des camps de concentration et d'extermination nazis, le témoignage pénètre directement la sphère publique, s'affranchissant de la discipline historique. Dans le cadre de cet article, nous allons tout d'abord nous employer à décrire cet objet sociodiscursif particulier qu'est le témoignage ${ }^{3}$, en essayant d'en définir les spécificités, dans une perspective essentiellement épistémologique et discursive. Nous verrons ensuite comment la mémoire de la Shoah, toute en ambiguïté et en paradoxe, peut s'énoncer et se transmettre, comment ses témoignages participent justement à la construction d'une mémoire collective, et enfin en quoi les récits des rescapés des camps de concentration et d'extermination nazis singularisent la problématique du témoignage et renouvellent en un certain sens la question des frontières entre les genres historiques et littéraires.

Le témoignage se définit primitivement comme un compte-rendu rétrospectif d'événements avec lesquels le locuteur a entretenu une relation perceptuelle directe et dont il se souvient. Cette première définition met d'emblée en jeu deux composantes essentielles du témoignage : sa dimension discursive et sa dimension indubitablement mémorielle ${ }^{4}$. Ces faits qui nous viennent du passé sont ainsi médiatisés d'une part par le souvenir - l'accès au passé transite par la mémoire - et d'autre part par une mise en récit, une narrativisation. Ces deux formes de médiatisation introduisent nécessairement des biais dans l'accès au passé. En outre, à l'instar de la triple identité de nom entre l'auteur,

1 Nous faisons bien entendu allusion aux victimes de la Solution Finale, dans les camps de concentration et d'extermination nazis.

2 Au sens de « retour à la conscience éveillée d'un événement reconnu comme ayant eu lieu avant le moment où celle-ci déclare l'avoir éprouvé, perçu, appris » (Ricœur $2000: 69$ ).

3 Le témoignage est un sujet très en vogue ces derniers temps. Il suffit de regarder le nombre de colloques et d'ouvrages récemment consacrés à ce sujet.

4 Pour Paul Ricœur (2000), le témoignage constitue la structure de transition privilégie entre mémoire et histoire. Dans le Fonds des enregistrements audiovisuels des témoignages des rescapés des camps de concentration et d'extermination nazis, recueilli par la Fondation Auschwitz, cet aspect est omniprésent, tant au niveau des témoignants (je me rappelle, je me souviens, etc.) que des interviewers (vous souvenezvous, avez-vous souvenance, etc.). Dans un des témoignages de ce fonds par exemple (celui de SW), 18 des interventions des interviewers sont introduites de la sorte. 
le narrateur et le personnage, prescrite par le pacte autobiographique (Lejeune 1996), nous poserons pour le témoignage celle de l'auteur (référent extratextuel qui en tant que signataire du témoignage en assume la pleine responsabilité et se porte garant de la vérité qu'il transmet), du témoignant (personne qui produit le témoignage, hic et nunc) et du témoin (personne qui a vécu les événements, in illo tempore) ${ }^{5}$. Témoigner, en tant qu'acte de discours, consiste donc à « affirmer une proposition en invoquant la position de témoin de l'état de choses représenté » (Vanderveken 1988 : 171).

Cette première contrainte est essentielle dans la mesure où la participation du locuteur à la situation qu'il décrit factualise ipso facto son récit. C'est le critère de certification autobiographique, qui constitue le noyau même du témoignage (Dulong 1988, VelcicCanivez 2002) et qui l'inscrit dans le registre du factuel, le soumettant du même coup au pacte référentiel : le témoignage doit apporter une information sur un objet ou un événement extratextuel, appartenant au monde réel et ayant vraiment existé ${ }^{\text {. De par sa }}$ nature référentielle, le témoignage est donc vériconditionnel, «soumis à l'alternative du vrai et du faux dans la logique de notre univers de référence » (Adam 1992 : 24), à l'inverse de l'énoncé fictionnel qui y échappe ${ }^{7}$. Cependant, contrairement au récit autobiographique, qui met l'accent sur la personne - auteur-narrateur-personnage -, sur son évolution et sur sa personnalité (Lejeune 1996), le témoignage se focalise sur les événements, à travers le regard particulier du témoin, qui n'est, par conséquent, qu'un instrument de savoir relégué au second plan (Goldschläger 1996).

Cette dernière spécificité du témoignage nous semble particulièrement intéressante au regard de la dimension vériconditionnelle des discours factuels. Dans le cas de l'autobiographie, le narrateur n'agit finalement que vis-à-vis de lui-même : « nous n'avons des comptes à rendre à personne de l'image de nous-mêmes. Nous le faisons, il est vrai, à nos risques et périls : l'oubli volontaire fait naître des remords, le refoulement de certains souvenirs conduit à la névrose. C'est l'intérêt de l'individu qui préside à la construction de cette image : elle aide à vivre un peu moins mal, elle contribue à son confort mental et à son bien-être. Personne n'a le droit de nous imposer l'image que nous avons de notre propre passé, même si nombreux sont ceux qui s'y essaient; en un certain sens, nos souvenirs sont irréfutables car ils comptent par leur propre existence, non par la réalité à laquelle ils renvoient » (Todorov 2000 : 141-142). Le témoignage, par contre, dans la mesure où il traite non plus exclusivement de l'individualité existentielle du témoin mais davantage des événements du monde auxquels ce dernier a pris part, entre dans la sphère publique et acquiert une portée collective ${ }^{8}$. Il ne peut dès lors pas prétendre seulement

5 Sur cette dissociation des instances énonciatives du témoignage, voir aussi Wisard 1994.

6 «L'une des conditions de l'accomplissement réussi de l'acte de langage de la référence est que l'objet auquel le locuteur fait référence doit exister » (Searle $1982: 115)$.

7 «L'énoncé de fiction n'est ni vrai ni faux (mais seulement, aurait dit Aristote, possible), ou est à la fois vrai et faux : il est au-delà ou en deçà du vrai et du faux, et le contrat paradoxal d'irresponsabilité réciproque qu'il noue avec son récepteur est un parfait emblème du fameux désintéressement esthétique » (Genette $1991: 20)$.

8 « Jurer, attester et témoigner, tout comme suggérer, prophétiser, vaticiner, assurer et rappeler, sont tous des actes de discours essentiellement dirigés vers un ou plusieurs allocutaires différents du locuteur. Conséquemment, tous ces actes de discours exigent un accomplissement public » (Vanderveken 1988 : 172). 
à une simple vérité du locuteur - une L-vérité pour reprendre la terminologie de Alain Berrendonner $(1981)^{9}$ - mais à une vérité en soi, une vérité universelle - une Ø-vérité -, qui engage la responsabilité du locuteur ${ }^{10}$.

Ainsi, il ne suffit pas que le témoignage soit attesté, il faut qu'il soit également ratifié, accrédité. Le témoignant demande à être cru et ses destinataires endossent par conséquent un rôle plus actif : celui de témoignaire accréditeur et juge. D'un point de vue épistémologique, le témoignage procède donc d'un double mouvement : premièrement, celui qui va de l'évidence liée à la perception des faits par le témoin ${ }^{11}$ à la vérité de leur mise en discours par le témoignant ${ }^{12}$ (Danblon 2004); et deuxièmement, celui qui va de la vérité du locuteur - ses croyances, ses convictions ${ }^{13}$ - à la vérité universelle. Ces deux mouvements correspondent en fait aux deux principes constitutifs du témoignage : la mise en discours d'une réalité perçue ou vécue et son accréditation ${ }^{14}$. Cependant, quelles sont les conditions de cette accréditation, entre la faillibilité du témoignage, expérimentalement mise en évidence par la psychologie qui mène inévitablement au scepticisme, et sa fiabilité présumée, qui découle tout naturellement de la créance spontanée accordée par défaut au propos d'un tiers?

Cette disposition à croire ce que les autres racontent - principe de crédulité, principe de charité interprétative - repose sur le principe de véracité (celle-ci étant comprise comme une propension de l'être humain à dire la vérité), qui repose lui-même sur la norme de sincérité (un locuteur L ne peut affirmer p que s'il croit que p est vrai) ${ }^{15}$. En

9 Quand on dit que $\mathrm{p}$ est L-vrai, cela revient à dire que $\mathrm{p}$ est vrai pour le locuteur et, in fine, que L croit que $\mathrm{p}$ est vrai.

Cette exigence de vérité est constitutive de l'acte de témoigner, sous l'influence des paradigmes judiciaire et historique. Le témoignage consiste en la « déclaration de ce que l'on a vu, entendu, perçu pour servir à l'établissement de la vérité » (Le Robert 2003 : 2580).

11 « Nous sommes le 12 avril 1945, le lendemain de la libération de Buchenwald. L'histoire est fraîche, en somme. Nul besoin d'un effort de mémoire particulier. Nul besoin d'une documentation digne de foi, vérifiée. C'est encore au présent, la mort. Ça se passe sous nos yeux, il suffit de regarder... La réalité est là, disponible. La parole aussi » (Semprun 1994 : 23).

«For inasmuch as the diarists and memoirists see themselves as traces of experiences, and their words as extensions of themselves, the link between words and events seems quite literally self-evident : that which has touched the writer's hand would now touch the reader (...). But for the reader with only words on a page, the authority for this link is absent. The words in a reproduced Holocaust diary are no longer traces of the crime, as they were for the writer who inscribed them; what was evidence for the writer at the moment he wrote is now, after it leaves his hand, only a detached and free-floating sign, at the mercy of all who would read and misread it. Once he withdraws from his words, the writer has in effect also withdrawn the word's evidentiary authority, the only link it ever had to its object in the world » (Young 1987: 413). Au sens de certitude subjective.

Cette accréditation, qui consiste finalement dans le passage de la vérité du locuteur à la vérité en soi, est essentielle et constitutive de l'acte même de témoigner. Le pacte testimonial, qui inclut cette dimension d'accréditation, excède donc le pacte autobiographique, au sens que lui donne Philippe Lejeune.

15 Cette norme constitue par ailleurs la condition de sincérité des actes discursifs assertifs, dont l'acte de témoigner. On la retrouve également au niveau des maximes gricéennes qui régissent nos comportements langagiers (maxime de qualité / de véridicité : Que votre contribution soit véridique : 1) n'affirmez pas ce que vous croyez être faux, 2) n'affirmez pas ce pour quoi vous manquez de preuves). Pour plus de détails en ce qui concerne le principe de charité interprétative, voir Davidson 2001, Reid 1983, Coady 1992. 
philosophie du langage, on s'est longtemps interrogé sur la légitimité du passage entre le principe de véracité et le principe de crédulité. Pour régler cet hiatus parfois un peu gênant ${ }^{16}$, on convoque généralement une condition supplémentaire : il est légitime que A [l'auditeur] accepte spontanément le statut véridique de p si, lorsque L [le locuteur] affirme $\mathrm{p}$ (ce qui signifie, selon la norme de sincérité, que L croit que p est vrai), L a des raisons de croire que $\mathrm{p}$ est vrai - en d'autres mots, nous avons des raisons de croire un locuteur qui dit $\mathrm{p}$ si nous avons des raisons de croire qu'il a des raisons de croire $\mathrm{p}^{17}$. A partir de là, l'accréditation accordée par l'auditeur est rationnellement justifiée et repose à la fois sur la fiabilité du locuteur et sur la vraisemblance des événements qu'il rapporte. Dans le cas des témoignages, la fiabilité du locuteur repose sur le fait qu'il a entretenu une relation perceptuelle - et/ou expérientielle - directe avec les événements qu'il décrit ${ }^{18}$. Tout au long de sa prise de parole, le témoignant ne cesse dès lors de réaffirmer, au moyen de marques linguistiques diverses, son statut de témoin.

Par contre, ce qui peut se révéler problématique dans le cas des témoignages de la Shoah tient au caractère particulièrement extrême - irréductible - et non partagé des expériences qui y sont décrites et sur la rupture cognitive et civilisationnelle qu'ils ont engendrée. A propos du travail d'appropriation du passé, Todorov distingue deux étapes complémentaires : il s'agit d'une part d'attester des événements du passé et d'autre part, de les rendre compréhensibles, interprétables. Les deux types de vérités qui en découlent sont la vérité d'adéquation, qui vise la « correspondance exacte entre le discours présent et les faits du passé » (Todorov $2000: 135$ ) et la vérité de dévoilement, qui permet de « saisir le sens d'un événement» (Todorov 2000 : 135). Dans le cas des témoignages de la Shoah, ces deux vérités sont quelque peu mises à mal. En effet, la question du lien entre les catégories du vrai et du vraisemblable ${ }^{19} \mathrm{~s}^{\prime} y$ pose avec une résonance toute particulière. Un des critères de validité factuelle du témoignage - et donc d'accréditation - consiste en sa plausibilité, «sa cohérence avec le stock de savoirs partagés sur ce qui peut arriver dans ce monde » (Dulong 1997 : 66), sa conformité avec notre système d'acceptation des croyances (Sosa 1994), en bref avec notre sens commun, qui nous pousse à croire que nous partageons un monde commun auquel nous faisons face collectivement (Pollner 1991). Or, ce qui pose justement problème dans le cas des témoignages de la Shoah, ce n'est pas leur véracité mais leur vraisemblance, puisque toute continuité référentielle ou

16 Car, comme le souligne à juste titre Keith Lehrer, il n’y a aucune corrélation directe et rationnelle entre ce que les gens croient être vrai et ce qui est - effectivement - vrai (Lehrer 1994).

17 Cette condition se retrouve également aux niveaux de la théorie des actes de langage de Vanderveken, puisqu'il ajoute une condition préparatoire à l'accomplissement des actes illocutoires assertifs : en plus de croire le contenu propositionnel de ce qu'il asserte (condition de sincérité), le locuteur doit aussi avoir des raisons de croire en la vérité de ce contenu propositionnel.

18 «Les rapports institutionnels, hiérarchiques, de pouvoir, qui forment le statut social de X sont, de ce point de vue, hautement pertinents : pour pouvoir parler au nom du réel, il convient que votre parole soit autorisée : $X$ doit donc être paré du prestige de la science [expert], être un témoin privilégié des événements affirmés («j’y étais ; donc, ce que je raconte est vrai (tout court) »), etc. » (Berrendonner 1981 : 66-67).

19 Le vrai, c'est ce qui est vrai, ce qui est réel, tandis que le vraisemblable, c'est ce qui paraît vrai (Lalande 1993 : 1224-1226). Selon Fransiska Louwagie (2003), le vrai relève de l'historiographie (au sens de vérité historique, vérifiable par l'opération historiographique) tandis que le vraisemblable est une catégorie littéraire. 
expérientielle entre le monde extérieur et la réalité des camps a été intentionnellement anéantie par les nazis, qui ne cessaient d'ailleurs de répéter aux déportés que de toute façon, s'ils arrivaient un jour à raconter leur histoire, on ne les croirait pas. De même, la réalité tragique des camps représente par définition une aporie de sens, qui provoque une impasse communicationnelle et qu'il est difficile de dénouer selon certains sous peine de la banaliser ${ }^{20}$. Le rescapé se trouve dès lors confronté à un vrai dilemme : comment va-t-il s'y prendre pour communiquer cet univers irrationnel et hors normes, qui dépasse totalement l'entendement et les cadres cognitifs de l'auditeur, avec lequel il ne partage sur ce point aucun repère? Quels procédés discursifs, quelles stratégies narratives et rhétoriques va-t-il utiliser pour ce faire ? Quel type de vérité s'emploie-t-il finalement à transmettre? Qu'est ce qui fait la différence entre son discours et celui de l'historien? Nous allons consacrer la suite de cet article à répondre à ces questions en retraçant très rapidement les modalités d'énonciation et de réception des témoignages de la Shoah et en insistant sur la tension inhérente à cette prise de parole.

Nous allons commencer par évoquer, à propos des témoignages de la Shoah, la problématique du silence. En effet, une grande partie de la littérature qui se rapporte à cette question fait allusion à la difficulté pour les rescapés de relater leur expérience. La nature extrême de l'expérience concentrationnaire rend problématique sa transmission. On évoque souvent à propos des témoignages de la Shoah (ou des témoignages des grandes catastrophes de manière plus générale) la problématique de l'ineffabilité, le phénomène du témoignage impossible, le mythe du silence ${ }^{21}$. Les rescapés sont eux-mêmes très diserts à ce propos et ont tour à tour analysé les difficultés de transmission sous l'angle de l'indicibilité ou de l'inaudibilité. Pour certains, la Shoah ne trouve pas de mots pour se dire $^{22}$. Elie Wiesel l'exprime en ces termes (Wiesel $1982: 12$ ) :

Les mots me paraissaient bêtes, usagés, inadéquats, anémiques ; je les désirais brûlants. Où dénicher un vocabulaire inédit, un langage premier ?

Les difficultés de communication seraient ainsi inhérentes au langage lui-même puisque ce sont les mêmes mots qui servent à décrire la réalité du camp et celle du dehors (Levi 1987 : 132) :

Nous disons faim, nous disons fatigue, peur et douleur, nous disons hiver, et en disant cela nous disons autre chose, des choses que ne peuvent exprimer les mots libres, créés par et pour des hommes libres qui vivent dans leurs maisons et connaissent la joie et la peine.

Comment combler la distance entre le langage ordinaire et cette expérience extraordinaire ? C'est la problématique de l'ineffable, de l'inénarrable, à laquelle se

20 « Vouloir comprendre le mal, c’est vouloir l'expliquer, et donc le fonder, le justifier ; expliquer la Shoah, la comprendre, c'est la relativiser, c'est-à-dire la justifier, c'est donc être comprise par elle » (Abécassis 2003$2004: 10)$

21 La Shoah : témoignage impossible (Goldschläger \& Lemaire 1998), Les voix du silence (Guilbau 2002), Exil de la parole : du silence biblique au silence d'Auschwitz (Neher 1970), Elie Wiesel : variation sur le silence (Cohen 1988), etc.

22 Il existe d'ailleurs un lexique propre à l'expérience concentrationnaire: musulman, organiser, kapo, canada, etc. 
surimpose très vite une seconde problématique, celle de l'inaudible et de l'incompréhensible (Semprun 1994 : 26- 27) :

On peut tout dire de cette expérience. Il suffit d'y penser. Et de s'y mettre. D'avoir le temps, sans doute, et le courage, d'un récit illimité, probablement interminable, illuminé - clôturé aussi, bien entendu - par cette possibilité de se poursuivre à l'infini. (...) Mais peut-on tout entendre, tout imaginer? Le pourra-t-on? En auront-ils la passion, la compassion, la rigueur nécessaire ? Le doute me vient, dès ce premier instant, cette première rencontre avec des hommes d'avant, du dehors venus de la vie -, à voir le regard épouvanté, presque hostile, méfiant du moins, des trois officiers.

Comment dès lors témoigner, entre cette indicibilité et cette incapacité à comprendre ? Face à cette double problématique, certains rescapés ont gardé le silence ${ }^{23}$. Cependant, ce thème du silence, lieu commun si souvent mentionné quand on évoque la Shoah, peut paraître paradoxal quand on sait que la grande majorité des rescapés ont pris la parole et qu'aucun autre événement historique n'a sans doute jamais suscité autant de témoignages (Wieviorka 1998, Waintrater 2003, Walter 2003). Robert Antelme parlera même d'hémorragie d'expression. Primo Levi explique ce phénomène par un devoir de mémoire ${ }^{24}$. Pour Annette Wieviorka, cet argument fallacieux - le silence des témoins lié au phénomène de l'indicibilité - a servi de prétexte, jusqu'aux années septante, pour justifier l'inertie de la mémoire collective de la déportation. Selon elle, il s'agirait moins d'un problème de volonté de témoigner que d'une non-réceptivité du public, liée aux contextes politiques et aux horizons d'attentes de nos sociétés aux lendemains de la guerre. Déjà dans les années cinquante, ces témoignages étaient trop nombreux pour être recensés exactement. Aujourd'hui, il en existe certainement des centaines de milliers sans qu'aucune bibliographie exhaustive ne les reprenne systématiquement (Wieviorka 1998). Face à cette masse de données, il est impossible de définir exactement et de manière univoque les témoignages de la Shoah, tant ces derniers divergent quant à leur forme, leur enjeu et les circonstances de la prise de parole.

Quoi qu'il en soit, les nombreux rescapés qui ont choisi de témoigner répondent à diverses motivations - judiciaires, historiques, mémorielles, morales, identitaires, thérapeutiques, pédagogiques, politiques, sociales, etc. En outre, pour témoigner, les rescapés ont eu recours à des formes diverses, entre le pôle documentaire - historique et le pôle fictionnel - romanesque. Les voies empruntées sont multiples et difficiles à catégoriser, entre les dépositions formelles formulées dans le cadre judiciaire, les témoignages historiques, les journaux intimes - réels ou rétrospectifs -, les récits de vie spontanés ou réalisés sous forme d'entretien, les autobiographies écrites, les essais, les romans, les pièces de théâtre, la poésie, etc. (Pollak \& Heinich 1986, Heinich 1998). Ces différentes modalités discursives sont d'ailleurs intimement liées aux différents contextes

23 Ceux qui ont refusé de parler ont souvent justifié leur démarche non seulement par les problèmes de transmission que nous venons de commenter, mais aussi en invoquant un certain respect envers les disparus et le risque de banaliser l'expérience de la Shoah. Ils ont aussi fait état de leur honte d'avoir survécu (Levi 1989) et de leur souci de ne pas raviver des souvenirs qu'ils essayaient à tout prix de refouler.

24 « J'ai porté à l'intérieur de moi cette impulsion violente et j'ai écrit tout de suite, dès mon retour. Tout ce que j'ai vu et entendu, il fallait m'en libérer. De plus, sur le plan moral, civil, politique, témoigner était un devoir » (Levi, cité dans Mertens 2003 : 14). 
de production des témoignages et à leurs modes de sollicitation. La distance entre la prise de parole et l'événement en question, ainsi que le contexte sociopolitique où elle prend place, auront une incidence certaine sur le discours du témoignant. Il serait donc pertinent d'évaluer les données historiques propres à la production et à la réception de ces témoignages.

Plusieurs auteurs se sont risqués à proposer des ébauches de périodisation, en repérant des cycles de recrudescence et d'accalmie (Goldschläger 1998, Hilberg 1994, Walter 1991, Walter 2001, Wieviorka 1992, Wieviorka 1995, Wieviorka 1998). A cet égard, il est très intéressant de retrouver le même rythme de publication pour les témoignages qui concernent la première et la seconde guerre mondiale : apparition des premiers témoignages dès le lendemain de la guerre, ralentissement, puis recrudescence plus ou moins une dizaine d'années après les événements (Prost 1977, Wieviorka 1998). Le désir de témoigner - ou plutôt le désir de mémoire - fut immédiat, contemporain à l'événement lui-même. En effet, les premiers témoignages émergent au cœur même de la guerre, sous la forme de fonds d'archives relativement élaborés et de journaux intimes individuels ${ }^{25}$. Les témoignages qui ont été produits dans l'immédiate après-guerre s'inscrivent dans un contexte particulier. Il s'agit soit de dépositions réalisées dans le cadre d'un procès, soit de témoignages spontanés qui s'expliquent par la pulsion intérieure ressentie par le rescapé pour témoigner de l'horreur, attester d'une réalité vécue, se libérer au plus vite de cette expérience tragique - et par là-même libérer la parole -, honorer des proches disparus, reconstituer son identité ou tout simplement pour réapprendre à vivre (Waintrater 2003). Ainsi, dès les lendemains de la guerre, de nombreuses commissions se sont constituées un peu partout pour récolter ces témoignage ${ }^{26}$. On crée aussi, dans la presse de cette époque, des sections entières dévolues à ces récits, des collections de livres, etc. De nombreux témoignages sont publiés ${ }^{27}$. Au début des années cinquante, les témoignages s'étiolent ${ }^{28}$. Il faudra attendre la fin des années cinquante, avec les publications de plusieurs témoignages phares $^{29}$ et le début des années soixante, avec le procès Eichmann, pour qu'émergent un véritable intérêt public pour le génocide et un mouvement de mémorisation. Le passage

25 Les archives des ghettos de Varsovie ou de Lodz sont les plus complètes. Par exemple, Emmanuel Ringelblum, avec l'aide d'autres, constitua ainsi une organisation de résistance (Oneg Shabbat) et entreprit de réunir et d'archiver des témoignages et d'autres documents matériels (affiches, ticket, lettres, presse, etc.) au cœur même du ghetto de Varsovie. Ces archives furent en partie retrouvées après la guerre et la liquidation du ghetto. On a également retrouvé des témoignages plus individuels, sous la forme de journaux intimes. L'Institut historique juif de Varsovie en conserve ainsi 321. Mais la plupart ont sans doute disparu en même temps que leurs auteurs.

26 C'est le cas par exemple de la Commission centrale d'histoire juive de Pologne qui recueillit, entre 1944 et 1948, plus de sept mille trois cents témoignages.

27 Rien qu'entre 1945 et 1948, Annette Wieviorka décompte pas moins de 114 ouvrages de témoignage publiés : 34 en 1945, 37 en 1946, 36 en 1947 et 7 en 1948 (Wieviorka 1992 : 168).

28 Ce qui ne signifie pas pour autant que la question du génocide tombe aux oubliettes. A cette époque, les derniers camps pour personnes déplacées ferment leurs portes et les Juifs qui attendaient d'être accueillis dans un pays ont pu émigrer, surtout aux Etats-Unis et en Israël. En 1951, le chancelier de la République fédérale allemande, Adenauer, endosse la responsabilité des crimes commis durant la seconde guerre mondiale et promet une réparation pour les préjudices causés.

29 L'espèce humaine de Robert Antelme en 1957, La Nuit d'Elie Wiesel en 1958, Le Dernier des Justes d'André Schwarz-Bart en 1959, etc. 
du temps y est également pour quelque chose car il a fallu du recul pour appréhender l'horreur de la Shoah et la décanter : «Elle (la volonté de témoigner) correspond à la sortie de sidération; les sociétés ont en effet besoin d'un temps de latence avant d'être capables d'affronter les blessures de l'histoire » (Waintrater 2003/2004 : 82).

En 1961, le procès Eichmann signe la statufication du témoin en termes d'identité sociale et comme détenteur d'histoire, et libère la parole testimoniale. En Europe, aux Etats-Unis ou en Israël, la mémoire du génocide est rendue publique ${ }^{30}$ et se revendique d'une certaine identité juive. Cette date marque pour Annette Wieviorka l'avènement de l'ère du témoin : "Pour que l'expression du souvenir du Hurbn pénètre le champ social, il faut que la configuration politique change, que le témoignage se charge d'un sens qui dépasse l'expérience individuelle, qu'il soit porté par des secteurs de la société. Ce sera chose faite avec le procès Eichmann, au prix d'une modification du contenu et de la signification de cette mémoire » (Wieviorka 1998 : 79). En effet, le procès Eichmann, diffusé internationalement, a eu un impact énorme sur la dynamique testimoniale. On y rencontre une volonté explicite de donner une leçon d'histoire et un désir marqué de transmission. Lors de ce procès, les témoignages de rescapés, appelés en masse à la barre, jouent un rôle prépondérant, au point d'en éclipser très vite le protagoniste principal : Eichmann lui-même. L'on cherche moins, finalement, à accumuler des preuves pour condamner Eichmann qu'à provoquer une réelle prise de conscience et toucher le cœur du public. Gidéon Hausner, qui fut le grand ordonnateur du procès, l'exprime en ces mots : « le seul moyen de faire toucher du doigt la vérité était d'appeler les survivants à la barre en aussi grand nombre que le cadre du procès pouvait l'admettre et de demander à chacun un menu fragment de ce qu'il avait vu et de ce qu'il avait vécu. Le récit d'un certain enchaînement de circonstances fait par un seul témoin est suffisamment tangible pour être visualisé. Mises bout à bout, les dépositions successives de gens dissemblables, ayant vécu des expériences différentes, donneraient une image suffisamment éloquente pour être enregistrée. Ainsi espérais-je donner au fantôme du passé une dimension de plus, celle du réel » (Hausner $1966: 384$ ).

Le procès de Jérusalem a initié un récit collectif et médiatisé de la Shoah et a amorcé un nouveau genre de témoignage, le témoignage pour mémoire, qui se distingue du témoignage judiciaire ou historique. Plutôt que de chercher des preuves, de vérifier l'authenticité des événements décrits, on valorise la dimension subjective du témoin, ses émotions et ses états d'âme, sa capacité à transmettre des expériences a priori non partagées avec le récepteur - quels que soient les moyens et stratégies qu'il déploie pour y parvenir - et le rôle que cette même transmission peut jouer dans la construction d'une mémoire collective. D'autres événements sociaux, politiques ou culturels viennent par la suite renforcer encore cette volonté de porter témoignage et le regain d'intérêt que le phénomène suscite, comme la Guerre des Six-Jours en 1967, l'émergence publique des premières théories révisionnistes à la fin des années septante (Rassinier, Faurisson, etc.), l'engouement pour les récits de vie de toutes sortes ${ }^{31}$ et la prolifération des témoignages

30 Hors des cercles privés de la famille ou des Amicales d'anciens déportés.

31 Si bien qu'en février 1982, le journaliste Frédéric Gaussen écrit dans Le Monde : « L'idée s'est imposée que toutes les vies se valent et sont bonnes à raconter». 
privés sur la scène publique ${ }^{32}$ (Lejeune 1980), la diffusion du feuilleton télévisé Holocauste en 1978, celle du film Shoah en 1985, les procès en France de Klaus Barbie en 1987, de Paul Touvier en 1994, de Maurice Papon en 1998 et bien sûr, à chaque fois, les anniversaires de la Libération. Il n'est dès lors plus question, pour beaucoup, de garder le silence ; c'est un devoir - pédagogique, civil - de porter témoignage, d'autant plus que certains témoins commencent inéluctablement à disparaître.

C'est dans ce contexte que s'organisent de manière plus structurée et institutionnalisée les différents milieux de mémoire et qu'apparaissent les premières campagnes de collectes systématiques de témoignages (Fortunoff Video Archive for Holocaust Testimonies en 1981, Survivors of the Shoah Visual History Foundation en 1994, sous 1'impulsion de Steven Spielberg à la suite du film La liste de Schindler). Le support du témoignage change : avec l'avancée des progrès techniques, on passe du papier au magnétophone et à la caméra. Il s'agit dès lors de donner la parole à tous les rescapés, y compris à ceux qui n'ont pas pris la plume ou qui n'ont même jamais pris la parole publiquement. A travers ces campagnes d'enregistrements, c'est l'accumulation des expériences individuelles qui est visée afin que se construisent un discours collectif et une mémoire sociale des événements. La fonction du témoignage a donc changé depuis la fin de la guerre. On ne cherche plus réellement à rendre compte et à démontrer les événements liés au génocide mais plutôt à les transmettre, à les partager. On est donc passé, en quelques décennies d'une mémoire occultée à une mémoire quasi saturée ${ }^{33}$, sacralisée ${ }^{34}$, qui pose alors la question du rapport entre mémoire et histoire, et celle des bienfaits liés à cette rémanence de la mémoire. Cependant, ces projets de collectes d'enregistrements audiovisuels ainsi que les nombreux témoignages publiés ne vont pas sans susciter des interrogations quant au statut des sources orales et aux relations qui s'instaurent entre le récit testimonial, forcément subjectif, et l'approche historiographique. Comment considérer le témoignage? Comment le traiter? Comment l'exploiter?

Les problèmes liés à l'utilisation des témoignages oraux et des récits de vie ont longtemps parasité les réflexions en sciences humaines (Bertaux 1997), que ce soit en sociologie (où le témoignage est vu en tant que mode d'investigation des conduites humaines et des processus sociaux), en psychologie ou en psychanalyse (à cause de la fonction exploratoire et analytique des récits de vie) et, bien entendu, en histoire. Plus que partout ailleurs, le problème du rapport entre la part de fictionnalité et la réalité indubitable des récits de vie se pose dans la discipline historique, qui remet d'ailleurs souvent en question le statut des sources orales (Wallenborn 2004). Dans quelle mesure les stratégies narratives peuvent-elles contribuer à l'écriture de l'histoire et à la restitution des événements historiques ? Comment s'articule la conviction subjective du témoignant et la vérité historique ? Ce débat est loin d'être entièrement tranché, et la tension entre témoins et historiens reste souvent perceptible (Combe 2003). Très fréquemment, le discours du témoignant interfère avec celui de l'historien, le concurrence même. Le

32 Pour François Hartog (2000), c'est l'ère de l'économie médiatique : le témoignage se fait en direct sur la scène publique, échappant par-là même aux historiens. 
premier reproche au second de parler de ce qu'il ne connaît pas réellement, tandis que le second regarde les témoignages avec circonspection, arguant de leur manque de fiabilité. Pour les historiens, entre l'expérience brute et sa mise en récit, il y a un pas ; la parole est loin d'être transparente. La critique historique met à nu les nombreux biais qui pèsent sur la perception de l'événement, sur sa mémorisation, sur sa réévaluation rétrospective nourrie des discussions et des lectures qui ont suivi -, sur le contexte énonciatif de la prise de parole (témoignage judiciaire, entretien, conférence, etc.) et, bien entendu, sur le mode de restitution (déposition, récit de vie, support écrit ou oral, etc.). Nombreux sont ceux qui pensent que ces données sont trop empreintes de subjectivité et trop remplies d'erreurs pour pouvoir guider notre appréhension des événements historiques ${ }^{35}$.

Quoi qu'il en soit, comme le fait remarquer à juste titre Todorov, les deux démarches sont complémentaires et correspondent à des visées différentes : « Si nous voulons connaître de l'intérieur les expériences des tenants d'idéologies opposées, nous ferions bien d'écouter le récit d'un milicien et celui d'un résistant. Si nous cherchons à estimer la valeur de ces positions, les conséquences pratiques de l'une et de l'autre, le rapport entre paroles et actes, nous ferions mieux de nous tourner vers les travaux des historiens. Si nous voulons disposer de dates, de chiffres, de noms propres, nous privilégierons l'enquête de l'historien; si nous cherchons à nous immerger dans le vécu des acteurs, le récit du témoin est irremplaçable. Si nous désirons comprendre le destin des déportés de Kolyma, nous n'avons pas à choisir entre l'analyse historique de Conquest et le témoignage de Guinzbourg, pas plus que nous ne sommes contraints d'opter pour Raul Hilberg et contre Primo Levi quand nous nous penchons sur Auschwitz » (Todorov 2000 : 144). La parole testimoniale pénètre donc le vécu des témoins selon une approche personnelle, intimiste, subjective tandis que le discours scientifique de l'historien permet une appréhension plus objective et globale. La mémoire de l'événement nécessite conjointement les deux modalités discursives.

Les récits de témoignage - et de manière paradigmatique ceux de la Shoah - sont donc un genre de discours tendu, hybride, ambivalent, aux confins à la fois des domaines de l'historiographie et de la littérature. A partir de l'Antiquité, la discipline historique s'est développée en s'affranchissant progressivement du domaine littéraire. La quête de la vérité, le souci d'objectivation sont devenues les visées principales de l'entreprise historienne, qui s'apparente en cela à l'enquête judiciaire. Au $20^{\mathrm{e}}$ siècle et notamment avec les écrits sur les camps de concentration et d'extermination nazis, nombre d'historiens - Paul Veyne, Michel de Certeau, Louis Mink ou encore Hayden White - se mettent à réfléchir aux modalités d'écriture de l'histoire et à leurs liens avec la fiction et la littérature. De même, le témoignage embrasse - et embrase - le champ littéraire puisqu'un nouveau genre est créé, malgré le paradoxe terminologique évident que cette conjoncture peut engendrer : il s'agit de la littérature de témoignage ou du témoignage littéraire, qui se confond presque intégralement avec ce que l'on appelle, d'autre part, littérature des camps, littérature concentrationnaire, littérature de la Shoah, etc. Cependant, dès qu'il est question de relater l'expérience extrême de la Shoah sous une forme un tant soit peu

35 «Les transcriptions des témoignages que j’ai examinées sont pleines d'erreurs dans les dates, les noms des personnes, et les endroits, et ils manifestent à l'évidence une mauvaise compréhension des événements euxmêmes. Certaines de ces dépositions peuvent davantage égarer le chercheur non averti que lui être utiles » (Dawidowicz 1981 : 177). 
fictionnelle, les voix s'élèvent. L'exploitation du génocide par l'art pose des problèmes éthiques et moraux. Comment les visées vériconditionnelles du témoignage peuvent-elles s'affilier à des ambitions esthétiques et littéraires ? Pour certain, la fiction - littéraire, cinématographique - entame la crédibilité du témoignage et le disqualifie en tant que tel. C'est que, nous l'avons vu ( $c f$. note 7), la fiction suspend la question du vrai ou du faux ainsi que la position morale du récepteur, ce qui est évidemment problématique dans le cas des témoignages sur la déportation. L'on en arrive même à récuser le statut de témoignage à certains récits de rescapés, au nom de l'exigence de vérité propre à ce type de discours.

Mais de quelle vérité parle-t-on alors ? Il faut dissocier, comme l'a fait Todorov, la vérité d'adéquation, objective, factuelle, référentielle, historique, de la vérité de dévoilement, personnelle, subjective voire même intersubjective, mémorielle, exemplaire, distinguer la vérité littérale de la vérité littéraire. Comme le fait remarquer Todorov, « un constat de fait est ou vrai ou faux. Une interprétation des faits peut être intenable, donc réfutable, mais elle ne possède pas, à l'autre extrême, un seuil supérieur » (Todorov 2000 : 136). Ces deux types de vérité sont différentes mais néanmoins complémentaires : « il n’y a pas qu'une seule vérité, pis (...) la vérité du vécu peut s'opposer à celle, minutieusement établie au travers de la reconstitution des faits, de l'historien, sans qu'aucune de ces vérités ne se trouve par là même invalidée » (Combe 2003 : 25). Un témoignage erroné n'est pas un témoignage falsifié ${ }^{36}$. Les erreurs, les réinterprétations, les approximations, les dérapages verbaux ${ }^{37}$ et les marques de subjectivité inhérentes au témoignage sont parfois plus révélatrices des réalités vécues par leur protagoniste que des données plus factuelles (Young 1987). Ce qui importe, c'est que le récit fasse sens. De ce fait, tout dépend également de la perspective à partir de laquelle on se place : soit on considère que les faits sont transparents, qu'ils parlent d'eux-mêmes et qu'il n'y a rien à ajouter, soit, au contraire, on part du postulat que les faits attestés ne sont pas, à eux seuls, toujours porteurs de sens.

Par ailleurs, la transmission des faits à l'état brut est-elle même possible ? « La langue serait-elle capable de transcender la littéralité de l'expérience afin de lui rendre toute l'ampleur de sa signification? Ce qui revient à dire : la langue serait-elle capable de transcender sa propre littéralité afin d'accéder à un mode d'expression (symbolique) qui irait droit à l'essence (la vérité) du réel que l'on cherche à représenter ?» (Grierson 2003 : 319). Certains rescapés ont adopté la position inverse : « Ne parviendront à cette

36 C'est à partir de la condition de sincérité qu'on peut faire la distinction entre le témoignage erroné et le témoignage falsifié : dans le cas du premier, L croit en p même si p est faux tandis que dans le cas du second, L ne croit pas en p. A ce propos, il serait peut-être intéressant d'analyser le texte de Benjamin Wilkomirski (1997), qui constitue un faux témoignage (Grierson 2001).

37 Pour ces raisons, l'historien - ainsi que tout chercheur en sciences sociales - qui travaille sur des sources orales ne peut se satisfaire de simples transcriptions orthographiques, qui ne reprennent que ce qui se dit dans le document audio ou audiovisuel (niveau verbal), en ajoutant même parfois des niveaux d'interprétation supplémentaires (corrections de l'oral en énoncés grammaticalement corrects, suppression des temps morts et des dérapages verbaux, ajout de ponctuation, etc.). De même, ce type de transcription ne reprend pas les niveaux suprasegmental (intonation, intensité, rythme, etc.) et paraverbal (position du corps dans l'espace, posture, gestes, mimique, regard, etc.), pourtant indispensables pour la compréhension et l'analyse du discours. Nous insistons donc ici avec vigueur sur la nécessité d'utiliser des conventions qui permettent d'atteindre un degré de précision adéquat dans la tâche de transcription. 
substance, à cette densité transparente que ceux qui sauront faire de leur témoignage un objet artistique, un espace de création. Ou de recréation. Seul l'artifice d'un récit maîtrisé parviendra à transmettre partiellement la vérité du témoignage. Mais ceci n'a rien d'exceptionnel : il en arrive ainsi de toutes les grandes expériences historiques » (Semprun 1994 : 23). C'est que parfois, les témoins ont besoin de dépasser le langage ordinaire et sa banalisation pour pouvoir communiquer ce qui transcende leur propre vécu. L'esthétisation, la fiction, l'écriture littéraire et romanesque, les stratégies narratives et rhétoriques peuvent ainsi participer à la transmission et au partage du vécu tragique et hors-normes des déportés - « la vérité essentielle de l'expérience » (Semprun 1994 : 136) et nous aider à mieux entendre, à mieux comprendre.

Les témoignages rassemblés lors des campagnes d'enregistrements des années quatre-vingt, et notamment ceux issus du Fonds des enregistrements audiovisuels des témoignages des rescapés des camps de concentration et d'extermination nazis de la Fondation Auschwitz, présentent l'ambivalence que nous venons de décrire. Ils sont en effet enchâssés dans un récit de vie qui suit l'ordre chronologique de la biographie du témoin et qui comporte, à ce titre, un avant-déportation (naissance, situation familiale, enfance, étude, activités professionnelles, etc.) et un après-déportation (retour à la libération, reprise de l'activité professionnelle, etc.), même si ce sont évidemment les expériences liées à la guerre et à l'univers concentrationnaire qui occupent l'essentiel de l'enregistrement et le cœur même du propos. De cette manière, l'on entend favoriser une lecture plus intime de la Shoah. Les témoignants doivent rapporter les événements tels qu'ils les ont vécus, selon une perspective interne et malgré les distorsions que cela peut engendrer ${ }^{38}$. En retraçant des destins individuels et en se focalisant sur les impressions personnelles et les émotions des témoins, l'on favorise l'empathie des récepteurs, nécessaire à la transmission de la mémoire et à la construction d'une mémoire collective : «Tout se passe comme si nos efforts de compréhension proprement historique avaient atteint leurs limites et qu'une autre modalité de compréhension devait surgir, en creusant dans la mémoire, pour compléter l'abîme qui nous sépare de l'innommable » (Thanassekos 1996 : 17). D’un autre côté, on observe dans ces témoignages la mise en place d'un réel dispositif d'attestation et de crédibilisation, à travers des marques discursives diverses. Il faudrait dès lors appréhender ces témoignages à la fois comme des sources de factualité et comme des ressources de sens (Zaccaï-Reyners 2004) et les évaluer à l'aune de ce qu'ils sont réellement, c'est-à-dire des témoignages pour mémoire.

Pour conclure, nous aimerions revenir sur les deux types de traces - matérielles et psychiques - et sur les deux principales modalités discursives disponibles - discours historique et témoignage - qui nous permettent d'appréhender le passé et dont nous parlions en début d'article. Ainsi, pour établir l'existence des chambres à gaz et des

38 Dans les enregistrements de la Fondation Auschwitz, les interviewers insistent d'ailleurs pour obtenir des témoignants des informations plus personnelles, plus subjectives.

Exemples :

- $\quad$ AH Starting Time 00.09.29: TH

AH Starting Time 00.53.19: TH

AH Starting Time 00.32.18: TH non non (.) tels que vous les avez vécus je veux dire ces types de :- (0.) de conflit

non (.) VOS réactions (0.) à la vision de tout cela (0.)

non non (.) heu:- heu:- pas au niveau

philosophique (.) c'est au niveau du VEcu (0.) heu :

(.) c'est ça qui m'intéresserait 
fours crématoires, plusieurs modalités probatoires sont convoquées. Jean-Claude Pressac (1993) - qui n'est pas historien de formation - a méticuleusement fouillé les archives de l'ancienne direction des constructions SS d'Auschwitz afin de réunir les preuves matérielles (documents, vestiges, etc.) qui démontrent la mise en place des instruments de la Solution Finale dans les camps d'Auschwitz-Birkenau. Il espère aboutir à « une reconstitution historique ainsi affranchie des témoignages oraux ou écrits, toujours faillibles et se contractant en sus avec le temps » (Pressac 1993 : 2). L'option inverse qui préside aux campagnes d'enregistrements audiovisuels - consiste à faire parler les témoins directs du drame, les rescapés. Mais là encore, il n'y a pas d'univocité et chaque témoignant a recours à des stratégies différentes pour rendre compte des événements du passé. Nous allons examiner plus spécifiquement trois enregistrements audiovisuels et montrer que leurs enjeux diffèrent parfois grandement. Il s'agit des témoignages de PR, de SW et de MN. Nous allons commencer pas un extrait ${ }^{39}$ du témoignage de PR.

PR / Starting Time 01.02.30 / Séquence des chambres à gaz :

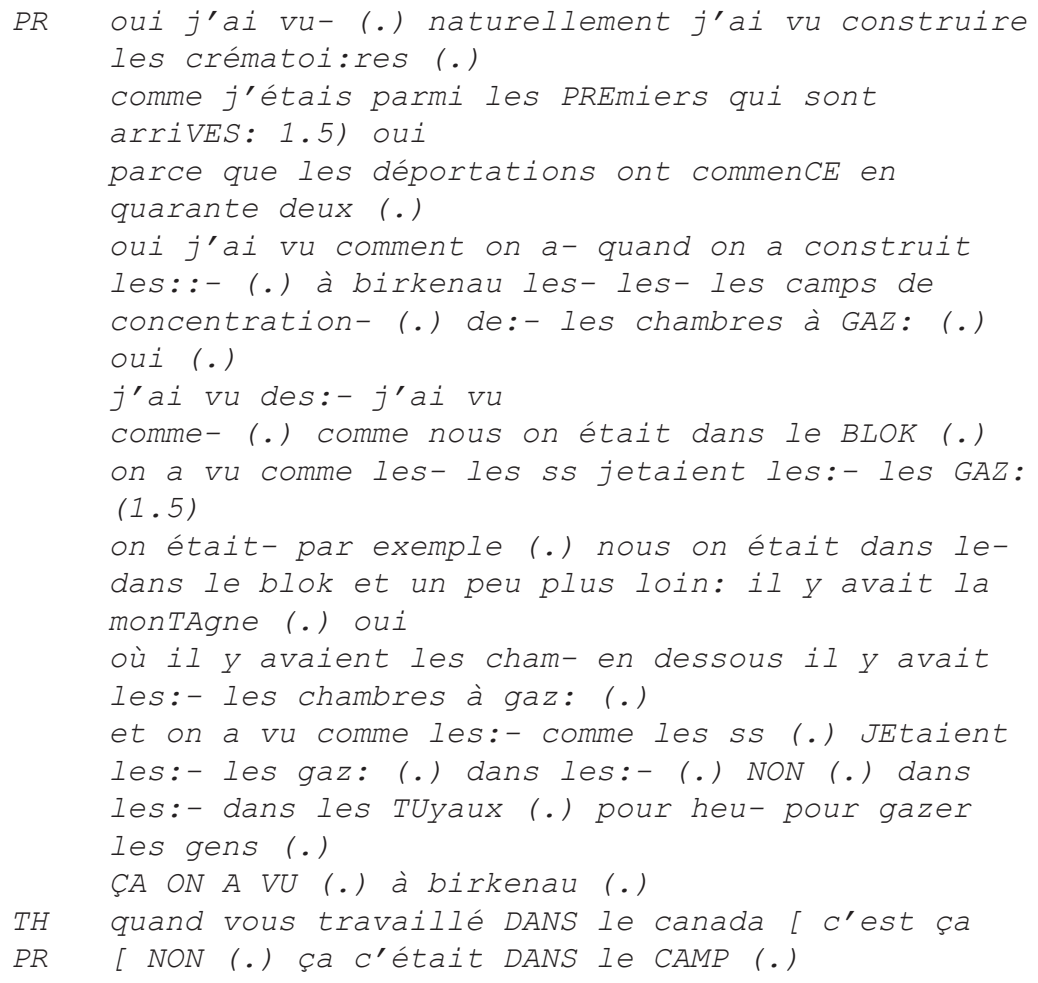

39 Nous utilisons la convention de transcription STAVIS (Balthasar 2001), qui comporte trois niveaux de transcription: le niveau segmental (propos du locuteur, pause, allongement de syllabe, syllabe accentuée, etc.), le niveau suprasegmental (intonation, intensité et rythme) et enfin le niveau paraverbal (positionnement du corps dans l'espace, posture, mimique, regard et gestes). Pour des raisons de lisibilité, les transcriptions reprises ci-dessous ne reprennent que le niveau segmental. Les pauses et les silences sont notées entre parenthèses, les syllabes accentuées par des majuscules et les allongements de syllabes par deux points (:). 


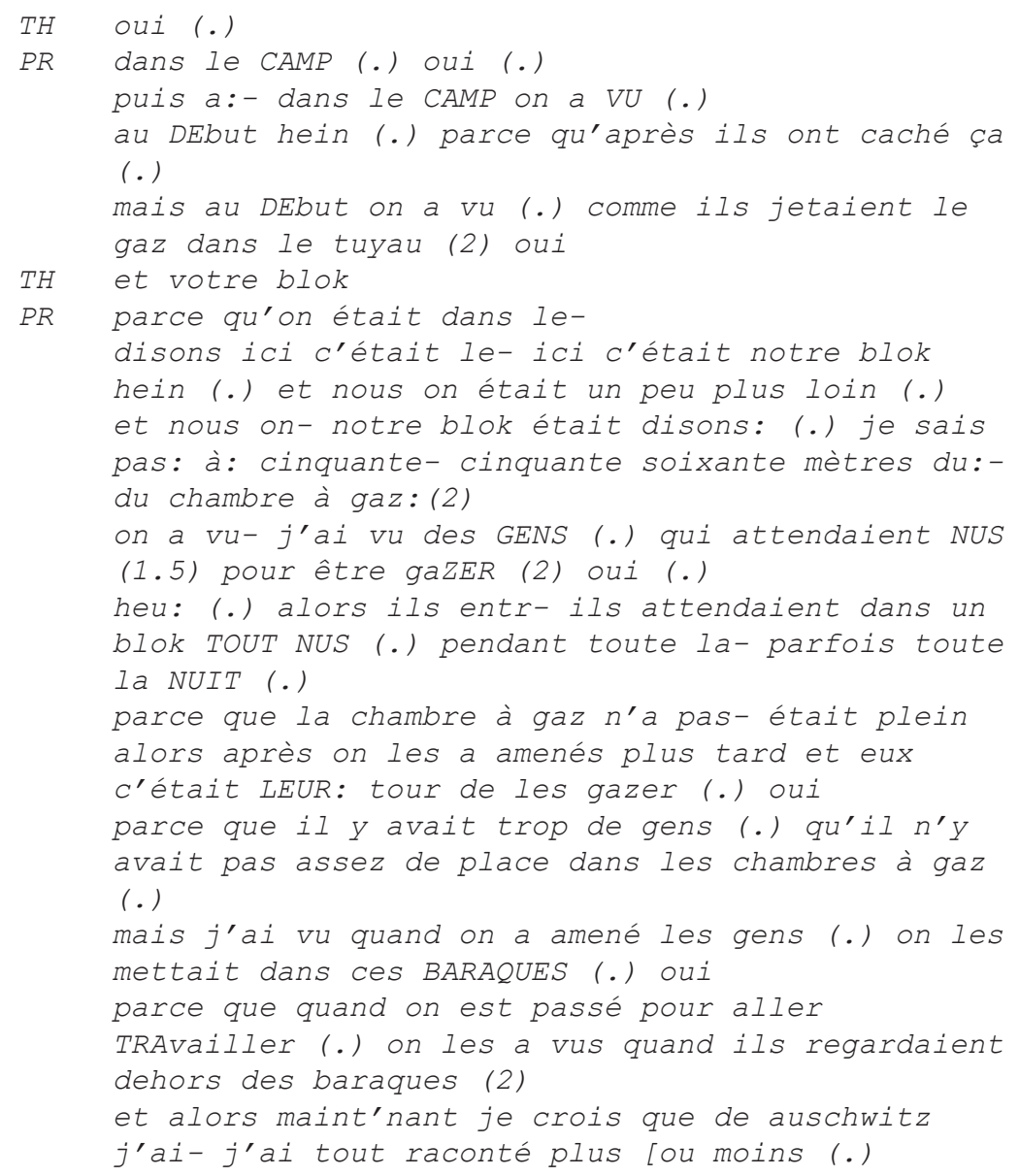

De manière très générale, le témoignage de PR est essentiellement descriptif et vise à attester des événements qu'il a vécus. En ce sens, il remplit parfaitement les fonctions définitoires du témoignage mentionnées plus haut. Dans cet extrait, le dispositif d'attestation est marqué discursivement, notamment au moyen des comptes-rendus de perception (j'ai vu / on $a v u$ ) - convoqués ici en masse par PR et qui lui permettent, en réaffirmant son statut de témoin, de légitimer la véracité de ses propos - ou des séquences en parce que - par lesquelles PR justifie a posteriori ses informations et les rend plus crédibles aux yeux de ses interlocuteurs. Cependant, ce témoignage présente par ailleurs deux difficultés majeures. En premier lieu, PR fait peu de récit et provoque peu d'empathie, ce qui peut générer des problèmes au niveau de la transmission du vécu et de son partage. En ce sens, son témoignage se rapproche davantage du témoignage judiciaire que du témoignage pour mémoire. En second lieu, le témoignage de PR se caractérise par une instabilité discursive, due à une mauvaise identification et gestion du format interactionnel dans lequel il est invité à produire son témoignage - interview plus formel que conversationnel. On ressent à l'écoute de son entretien une très nette forme de malaise et de tension qui résulte du fait que PR souffre du comportement neutre et peu réactif des interviewers - comportement par ailleurs tout à fait naturel compte-tenu 
de leur rôle institutionnel. Cette instabilité discursive et les tentatives d'attestation et de surjustification de PR peuvent se révéler parfois problématiques dans la mesure où elles peuvent être interprétées comme des sources de discrédit.

Les extraits des témoignages de $\mathrm{SW}$ et de $\mathrm{MN}$ que nous allons présenter ici sont assez différents. De manière générale, leur parole se situe dans un registre beaucoup plus symbolique, voire poétique parfois. Pour témoigner de l'existence des fours crématoires, ils mobilisent le potentiel évocatif de deux expériences perceptuelles relativement ordinaires : celle du ciel rouge ${ }^{40}$ chez SW et celle des effluves chez MN.

SW / Starting Time 01.08.19 / Séquence du ciel rouge (1) :

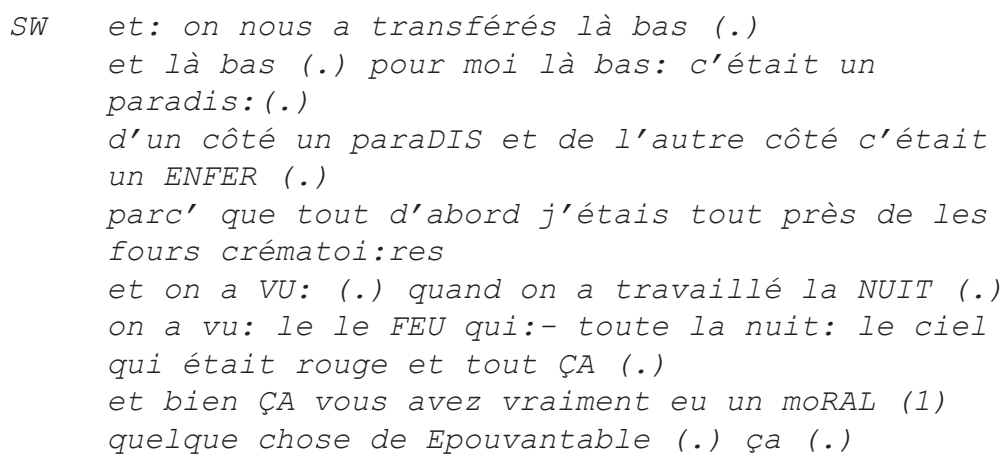

Quelques minutes plus tard, elle reprend la même expression :

SW / Starting Time 01.12.40 / Séquence du ciel rouge (2) :

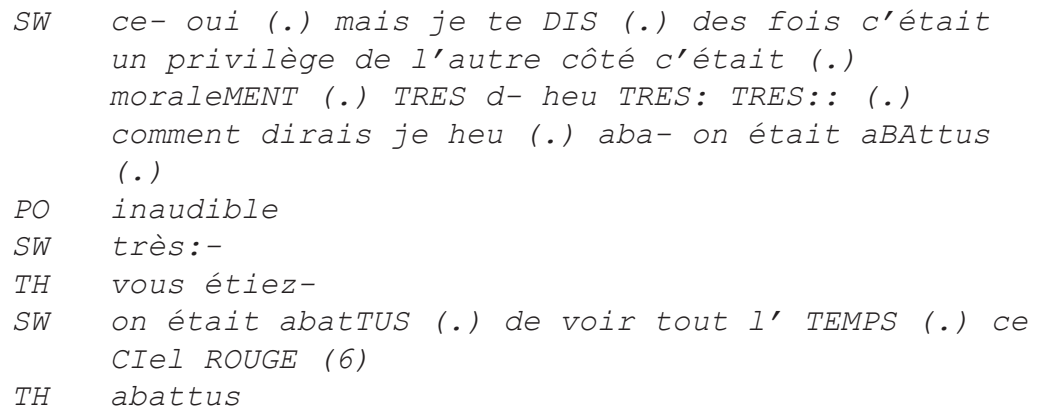

MN / Starting Time 02.44.31 / Séquence des effluves des chambres à gaz

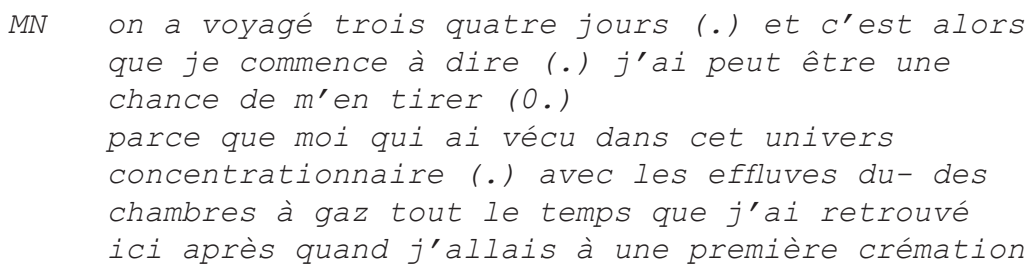

40 Métaphore souvent utilisée dans les textes littéraires et poétiques. 


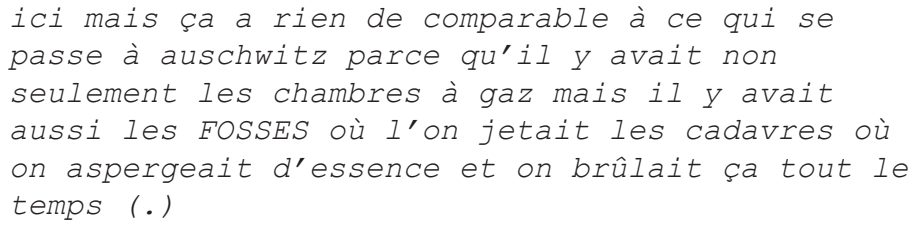

Dans ces trois extraits, l'on se rapproche du paradigme du témoignage pour mémoire, où il s'agit davantage d'évoquer et de transmettre que de prouver. Mais l'utilisation de ces stratégies risque de nuire à la valeur documentaire du témoignage au profit de sa valeur littéraire. D'ailleurs, on les retrouve dans les témoignages dits littéraires, notamment dans L'écriture ou la vie de Jorge Semprun où l'auteur évoque les chambres à gaz et les fours crématoires à partir des mêmes expériences perceptuelles de la fumée et de l'étrange odeur :

Le crématoire s'est arrêté hier, leur dis-je. Plus jamais de fumée sur le paysage. Les oiseaux vont peut-être revenir! Ils font la grimace, vaguement écœurés. Mais ils ne peuvent pas vraiment comprendre. Ils ont saisi le sens des mots, probablement. Fumée : on sait ce que c'est, on croit savoir. Dans toutes les mémoires d'homme il y a des cheminées qui fument. Rurales à l'occasion, domestiques : fumées des lieuxlares. Cette fumée-ci, pourtant, ils ne savent pas. Et ils en sauront jamais vraiment. Ni ceux-ci, ce jour-là, ils ne peuvent pas imaginer, quelles que soient leurs bonnes intentions. Fumée toujours présente, en panaches ou volutes, sur la cheminée trapue du crématoire de Buchenwald, aux abords de la baraque administrative du service du travail, l'Arbeitsstatistik, où j'avais travaillé cette dernière année. Il me suffisait d'un peu pencher la tête, sans quitter mon poste de travail au fichier central, de regarder par l'une des fenêtres donnant sur la forêt. Le crématoire était là, massif, entouré d'une haute palissade, couronné de fumée (Semprun 1994 : 22-23).

Etrange odeur », a écrit Léon Blum. Déporté en avril 1943, avec George Mandel, Blum a vécu deux ans à Buchenval. Mais il était enfermé en dehors de l'enceinte proprement dite du camp : au-delà de la barrière de barbelés électrifiés, dans une villa du quartier des officiers S.S. Il n'en sortait jamais, personne n'y pénétrait que les soldats de garde. Deux ou trois fois, il avait été conduit chez le dentiste. Mais c'était en voiture, la nuit, sur des routes désertes dans la forêt de hêtres. Les S.S., a-t-il consigné dans ses souvenirs, circulaient sans cesse mitraillette en bandoulière et chiens en laisse, dans l'étroit chemin de ronde ménagé entre la palissade barbelée et la maison. "Comme des ombres impassibles et muettes », a écrit Léon Blum. C'est la rigueur de cette clôture qui explique son ignorance. Léon Blum ne savait même pas où il se trouvait, dans quelle région de l'Allemagne il avait été déporté. Il a vécu deux ans dans une villa du quartier des casernes S.S. de Buchenwald en ignorant tout de l'existence du camp de concentration, si proche pourtant. " Le premier indice que nous en avons surpris, a-t-il écrit au retour, est l'étrange odeur qui nous parvenait souvent le soir, par les fenêtres ouvertes, et qui nous obsédait la nuit entière quand le vent continuait à souffler dans la même direction : c'était l'odeur des fours crématoires ». On peut imaginer Léon Blum, ces soirs-là. De printemps, probablement : fenêtres ouvertes sur la douceur du printemps revenu, les effluves de la nature. Moments de nostalgie, de vague à l'âme, dans la déchirante incertitude du renouveau. Et soudain, porté par le vent, l'étrange odeur. Douceâtre, insinuante, avec des relents âcres, proprement écœurants. L'odeur insolite, qui s'avérerait être celle du four crématoire. Etrange odeur, en vérité, obsédante. Il suffirait de fermer les yeux, encore aujourd'hui. Il suffirait non pas d'un effort, bien au contraire, d'une distraction de la mémoire remplie à ras bord de balivernes, de bonheurs insignifiants, 
pour qu'elle réapparaisse. Il suffirait de se distraire de l'opacité chatoyante des choses de la vie. Un bref instant suffirait, à tout instant. Se distraire de soi-même, de l'existence qui vous habite, vous investit obstinément, obtusement aussi : obscur désir de continuer à exister, de persévérer dans cette obstination, quelle qu'en soit la raison, la déraison. Il suffirait d'un instant de vraie distraction de soi, d'autrui, du monde : instant de non-désir, de quiétude d'en deçà de la vie, où pourrait affleurer la vérité de cet événement ancien, originaire, où flottait l'odeur étrange sur la colline de l'Ettersberg, patrie étrangère où je reviens toujours (Semprun 1994 : 16-17).

Le témoignage, et plus particulièrement celui des rescapés des camps de concentration et d'extermination nazis, est une parole génériquement tendue. Parce qu'elle doit comporter simultanément une dimension testimoniale et une dimension autobiographique (récit à la première personne). Parce qu'elle doit se situer entre une dimension singulière, individuelle (je) et une dimension collective et publique (nous, on). Parce qu'elle reproduit le paradoxe inhérent et si souvent invoqué du dicible et de l'indicible, du devoir de mémoire et de l'impasse communicationnelle. Parce qu'elle doit faire comprendre et faire accepter une réalité transcendantale et irréductiblement invraisemblable. Parce qu'elle doit exister, persister, entre l'exigence de dire le vrai - l'exacte vérité comme réalité -, l'incapacité du langage à reproduire la réalité extratextuelle stricto sensu et la nécessité de faire sens et de transmettre. Parce que le concept de vérité y est lui-même tendu, entre la vérité factuelle, objective et la vérité subjective. Et enfin, parce que cette parole testimoniale s'inscrit à l'intersection des genres historiques et littéraires et qu'elle nous oblige ainsi à nous interroger sur les limites et sur l'essence de ces deux modalités discursives. 


\section{Références bibliographiques}

AbecAssis, Eliette, décembre 2003-janvier 2004. «Peut-on parler de la Shoah?», Le Nouvel Observateur, pp. 10-13.

AdAm, Jean-Michel, 1992. Les textes : types et prototypes. Paris, Nathan.

Antelme, Robert, 1957. L'espèce humaine. Paris, Gallimard.

Balthasar, Lukas, 2001. Interaction audiovisuelle, théorie pragma-linguistique et transcription. Thèse de doctorat : Ecole des Hautes Etudes en Sciences Sociales (Paris) (2 vols).

Berrendonner, Alain, 1981. Eléments de pragmatique linguistique. Paris, Editions de Minuit.

Bertaux, Daniel, 1997. Les récits de vie. Perspective ethnosociologique. Paris, Nathan.

Certeau, Michel de, 1975. L'écriture de l'histoire. Paris, Gallimard.

CoAdy, Tony, 1992. Testimony. A philosophical Study. Oxford, Oxford University Press.

CoHen, Myriam, 1988. Elie Wiesel : Variations sur le silence. La Rochelle, Editions Rumeur des Ages.

Combe, Sonia, 2003. « Témoins et historiens : pour une réconciliation », in Jean-François Chiantaretto et Régine Robin (dir.), Témoignage et écriture de l'histoire, pp. 19-31. Paris, L'Harmattan.

Coquio, Catherine, 2000. «La vérité du témoin comme schisme littéraire », in Daniel Dobbels et Dominique Moncond'Hui (dir.), Les camps et la littérature. Une littérature du $20^{e}$ siècle, pp. 55-79. Poitiers, La Licorne.

DAnBlon, Emmanuelle, 2004. «Le témoignage : vérité ou évidence ? », in Jean-Philippe Schreiber, Adrien Antoniol, Barbara Pirlot et Hélène Wallenborn (dir.) Oralités. Les usages de la parole des gens en sciences humaines, pp. 53-66. Actes du séminaire Prosoposée I, Bruxelles, Université Libre de Bruxelles (Unité de recherche Sources audiovisuelles en histoire contemporaine).

DAVIDSON, Donald, 2001. Inquiries into Truth and Interpretation. Oxford, Clarendon Press.

Dawidowicz, Lucy S., 1981. The Holocaust and the Historians, Cambridge, Mass. et Londres, Harvard University Press.

Dulong, Renaud,

1997. « Les opérateurs de factualité. Les ingrédients matériels et affectuels de l'évidence historique », Politix 39, pp. 65-85.

1988. Le témoin oculaire. Les conditions sociales de l'attestation personnelles. Paris, Editions de l'Ecole des Hautes Etudes en Sciences Sociales.

Genette, Gérard, 1991. Fiction et diction. Paris, Seuil.

GolDSCHLÄGER, Alain, 1996. « La littérature de témoignage dela Shoah. Direl'indicible-lirel'incompréhensible », Texte : revue de critique et de théorie littéraire 19-20 (Le narratif hors de soi), pp. 259-278.

GoldschläGer, Alain et Jean-Charles Lemaire, 1998. La Shoah : témoignage impossible, Bruxelles, Editions de l’Université libre de Bruxelles.

Grierson, Karla,

2001. « Identité individuelle, identité collective : quelle vérité pour le récit de vie ? », in Philippe Forest et Claude Gaugain (dir.), Les romans du je, pp. 247-269. Nantes, Editions Pleins Feux.

2003. «Indicible et incompréhensible dans le récit de déportation », in Marie-Thérèse Mathet (dir.), L'incompréhensible. Littérature, réel, visuel, pp. 311-353. Paris, L'Harmattan.

Guilbau, Brigitte, 2002. Les voix du silence. Bruxelles, Editions Luc Pire.

Hartog, François, 2000. « Le témoin et l'historien », Gradhiva 27, pp. 1-14.

Hausner, Gidéon, 1966. Justice à Jérusalem. Eichmann devant ses juges. Paris, Flammarion. 
HeInICH, Nathalie, 1998. « Le témoignage, entre autobiographie et roman : la place de la fiction dans les récits de déportation », Mots 56, pp. 33-49.

Hilberg, Raul, 1994. La politique de la mémoire. Paris, Gallimard.

LALANDE, André, 1993. Vocabulaire technique et critique de la philosophie. Paris, PUF (Quadrige), (2 vols).

LeJEune, Philippe,

1980. Je est un autre. L'autobiographie, de la littérature aux médias, Paris, Seuil.

1996. Le pacte autobiographique. Paris, Seuil.

LeHrer, Keith, 1994. «Testimony, Justification and Coherence », in Matilal Bimal Krishna \& Chakrabarti Arindam (éds.), Knowing from Words. Western and Indian Philosophical Analysis of Understanding and Testimony, pp. 51-58. Dordrecht-Boston-Londres, Kluwer Academic Publishers.

LeVI, Primo,

1987. Si c'est un homme. Paris, Julliard.

1989. Les naufragés et les rescapés. Quarante ans après Auschwitz. Paris, Gallimard.

Louwagie, Franciska, 2003. «Une poche interne plus grande que tout. Pour une approche générique du témoignage des camps », Questions de communication 4, pp. 365-379.

Mertens, Pierre, 2003. Ecrire après Auschwitz? Semprun, Levi, Cayrol, Kertész, Paris, La Renaissance du Livre.

MinK, Louis, 1979. « History and Fiction as Modes of Comprehension », New Literary History 1, pp. 541-558.

NeHER, André, 1970. L'exil de la parole : du silence biblique au silence d'Auschwitz. Paris, Seuil.

Novick, Peter, 2001. L'Holocauste dans la vie américaine. Paris, Gallimard.

Pollak, Michaël et Nathalie HeInich, 1986. «Le témoignage », Actes de la recherche en sciences sociales pp. 62-63,

Pollner, Melvin, 1991. «Que s’est-il réellement passé ? Evénement et monde commun », in Jean-Luc Petit (dir.), L'événement en perspective, pp. 75-96. Paris, Editions de l'Ecole des Hautes Etudes en Sciences Sociales.

Pressac, Jean-Claude, 1993. Les crématoires d'Auschwitz. La machinerie du meurtre de masse. Paris, Presses du CNRS.

Prost, Antoine, 1977. Les Anciens Combattants et la Société française, 1914-1939. Paris, Presses de la FNSP, (3 vols).

REID, Thomas, 1983. "An Inquiry into the Human Mind on the Principles of Common Sense", in William Hamilton (éd.), The Works of Thomas Reid, pp., Hildesheim, G. Olms Verlagsbuchhandlung.

Riceur, Paul,

1972. «L'herméneutique du témoignage », in E. Castelli (dir.), Le témoignage. Actes du colloque organisé par le Centre international d'études humanistes et par l'Institut d'études philosophiques de Rome, pp. 35-61. Paris, Aubier.

2000. La mémoire, l'histoire, l'oubli. Paris, Seuil,

Schwarz-Bart, André, 1959. Le Dernier des Justes. Paris, Seuil.

SEARle, John R., 1982. Sens et expression. Etudes de théorie des actes du langage. Paris, Editions de Minuit.

SEMPrun, Jorge, 1994. L'écriture ou la vie, Paris, Gallimard.

SosA, Ernest, 1994. "Testimony and coherence», in Matilal Bimal Krishna et Chakrabarti Arindam (éds.), Knowing from words. Western and Indian Philosophical Analysis of Understanding and Testimony, pp. 59-67. Dordrecht-Boston-Londres, Kluwer Academic Publishers. 
Thanassekos, Yannis, 1996. «Préface», in Du Témoignage audiovisuel, Deuxième Rencontre Audiovisuelle Internationale sur le témoignage des survivants des camps de concentration et d'extermination nazis, pp. 17-21. Paris-Bruxelles, Fondation Auschwitz et Fondation pour la Mémoire de la Déportation.

ToDorov, Tzvetan,

1995. Les abus de la mémoire. Paris, Arléa.

2000. Mémoire du mal, tentation du bien. Enquête sur le siècle. Paris, Robert Laffont.

Vanderveken, Daniel, 1988. Les actes de discours : essai de philosophie du langage et de l'esprit sur la signification des énonciations. Bruxelles, Mardaga.

Velcic-Canivez, Mirna, 2002. « Le récit de témoignage comme genre. Spécificités référentielles », in Michel Ballabriga (dir.), Analyse des discours. Types et genres : communication et interprétation, pp. 317-328. Toulouse, Editions Universitaires du Sud (Collection Champs du Signe).

VEYnE, Paul, 1971. Comment on écrit l'histoire, Paris, Seuil.

WALlenborn, Hélène, 2004. L'historien, la parole des gens et l'histoire. L'exemple d'un fonds de témoignages audiovisuels de survivants des camps nazis. Thèse de doctorat, Université Libre de Bruxelles.

WALTER, Jacques, 2003. « Cadres du témoignage historique et médiatique, frontières disciplinaires », Questions de communication 3, pp. 11-30.

WAINTRATER, Régine,

2003. Sortir du génocide : Témoigner pour réapprendre à vivre. Paris : Payot.

décembre 2003-janvier 2004. « Le pacte testimonial », Le Nouvel Observateur, pp. 82-83

Whiте, Hayden, 1987. The Content of the Form: Narrative Discourse and Historical Representation. Baltimore, Johns Hopkins University Press.

WIESEL, Elie,

1958. La Nuit. Paris, Editions de Minuit.

1982. Paroles d'étranger : textes, contes et dialogues. Paris, Seuil.

Wieviorka, Annette,

1992. Déportation et génocide. Entre la mémoire et l'oubli. Paris, Plon.

1998. L'ère du témoin. Paris, Plon.

WiLkomirski, Benjamin, 1997. Fragments. Une enfance 1939-1948. Paris, Calmann-Lévy.

WisARD, François, 1994. L'énonciation d'un témoignage (L'étrange défaite de Marc Bloch). Lausanne, Bulletin de l'Institut de Linguistique et des Sciences du Langage de la Faculté de l'Université de Lausanne.

Young, James, 1987. "Interpreting Literary Testimony : A Preface to Rereading Holocaust Diaries and Memoirs”, New Literary History 2, pp. 403-423.

ZACCAÏ-ReYNERS, Nathalie, 2004. «Les paroles ont-elles un sens ? De quelques apports de l'herméneutique au questionnement épistémologique sur l'usage de l'entretien en sciences humaines », dans Oralités. Les usages de la parole des gens en sciences humaines. Actes du séminaire Prosoposée I, textes réunis par Jean-Philippe Schreiber, Adrien Antoniol, Barbara Pirlot et Hélène Wallenborn, Unité de recherche Sources audiovisuelles en histoire contemporaine, Bruxelles, Université Libre de Bruxelles, pp. 33-39. 
